\title{
Mixed $Q_{5} \mathbf{S}_{6}, \mathbf{S}_{7} \mathbf{O}_{8}, \mathbf{N}_{9} \mathbf{D}_{10}$ functional equations
}

\author{
M. Arunkumar ${ }^{1}$ and P. Agilan ${ }^{2 *}$
}

$$
\begin{aligned}
& \text { Abstract } \\
& \text { In this paper, the authors introduce and establish the stability of sing } \\
& \text { and nonic-decic functional equation of the form } \\
& \qquad \begin{array}{r}
\vartheta(2 p)=48 \vartheta(p)+16 \vartheta(-p) ; \\
\qquad(2 q)=192 \eta(q)+64 \eta(-q) ; \\
\psi(2 r)=768 \psi(r)+256 \psi(-r) ;
\end{array}
\end{aligned}
$$

In this paper, the authors introduce and establish the stability of single variable mixed quintic-sextic, septic-octic

in Banach space with the help of Ulam - Hyers and Radus methods.

\section{Keywords}

Quintic, sextic, septic,octic, nonic,decic functional equations, generalized Hyers - Ulam - Rassias stability.

AMS Subject Classification: 39B52, 32B72, 32B82.

${ }^{1}$ Department of Mathematics, Government Arts College, Tiruvannamalai - 606 603, TamilNadu, India.

${ }^{2}$ Department of Mathematics, Easwari Engineering College, Ramapuram, Chennai - 600 089, TamilNadu, India.

*Corresponding author: ${ }^{*}$ annarun2002@gmail.com; ${ }^{2}$ agilram@gmail.com

Article History: Received 22 November 2018; Accepted 09 May 2019

\section{Contents}

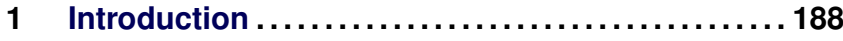

2 Stability Results: Direct Method : Odd Case ..... 189

3 Stability Results : Even Case ................. 191

4 Stability Results : Mixed Case ................ 192

5 Stability Results : Fixed Point Method: Odd Case194

6 Stability Results: Even Case.................199

7 Stability Results: Mixed Case $\ldots \ldots \ldots \ldots \ldots \ldots \ldots 200$

References ..............................201

\section{Introduction}

The source for the development of stability of functional equations is the question solicited by Ulam [28]. Hyers [16] presented an excellent answer to the question of Ulam. Later, the result of Hyers was generalized and refined further by many great mathematicians like Aoki [2], Th.M. Rassias [14], J.M. Rassias [27] and Gavruta [13] in various directions. The progress of the theory of stability of various types of functional equations such as quadratic, cubic, quartic, quintic, sextic, septic, octic, nonic, decic, undecic, duodecic, tredecic, quattordecic have been dealt by many mathematicians and there are lot of interesting and significant results available in the literature.

The stability problems of the following functional equations have been extensively investigated by a number of authors and there are many interesting results concerning this problem (see [3, 21, 29, 30]).

Recently, M.Arunkumar et.al., test the generalized Ulam - Hyers stability of the additive-quadratic and cubic-quartic functional equations

$$
f(2 x)=3 f(x)+f(-x)
$$

$$
g(2 x)=12 g(x)+4 g(-x)
$$

via Quasi-Beta Banach space and Intuitionistic fuzzy Banach space using direct and fixed point methods.

In this paper, the authors introduce and establish the stability of sigle variable mixed quintic-sextic, septic-octic and nonic-decic functional equation of the form

$$
\begin{aligned}
& \vartheta(2 p)=48 \vartheta(p)+16 \vartheta(-p) \\
& \eta(2 q)=192 \eta(q)+64 \eta(-q)
\end{aligned}
$$




$$
\psi(2 r)=768 \psi(r)+256 \psi(-r)
$$

in Banach space with the help of Ulam - Hyers and Radus methods.

Hereafter through out this paper, let us consider $X$ and $Y$ to be a normed linear space and a Banach space, respectively.

\section{Stability Results: Direct Method : Odd Case}

In this section, the generalized Ulam-Hyers stability of the functional equation (1.3), (1.4) and (1.5) odd case is investigated.

Theorem 2.1. Let $j \in\{-1,1\}$ and $\xi, \zeta, \varsigma: X \rightarrow[0, \infty)$ be a function such that

$$
\begin{aligned}
& \lim _{n \rightarrow \infty} \frac{\xi\left(2^{n j} p\right)}{2^{5 n j}}=0 \\
& \lim _{n \rightarrow \infty} \frac{\zeta\left(2^{n j} q\right)}{2^{7 n j}}=0 \\
& \lim _{n \rightarrow \infty} \frac{\zeta\left(2^{n j} r\right)}{2^{9 n j}}=0
\end{aligned}
$$

for all $p, q, r \in X$. Let $\vartheta, \eta, \psi: X \rightarrow Y$ be an odd function satisfying the inequality

$$
\begin{gathered}
\|\vartheta(2 p)-48 \vartheta(p)-16 \vartheta(-p)\| \leq \xi(p) \\
\|\eta(2 q)-192 \eta(q)-64 \eta(-q)\| \leq \zeta(q) \\
\|\psi(2 r)-768 \psi(r)-256 \psi(-r)\| \leq \varsigma(r)
\end{gathered}
$$

for all $p, q, r \in X$. Then there exists a unique quintic, septic,nonic mapping $Q_{5}, S_{7}, N_{9}: X \rightarrow Y$ and satisfying the functional equation (1.3), (1.4) and (1.5) such that

$$
\begin{aligned}
& \left\|\vartheta(p)-Q_{5}(p)\right\| \leq \frac{1}{32} \sum_{k=\frac{1-j}{2}}^{\infty} \frac{\xi\left(2^{k j}\right)}{2^{5 k j}} \\
& \left\|\eta(q)-S_{7}(q)\right\| \leq \frac{1}{128} \sum_{k=\frac{1-j}{2}}^{\infty} \frac{\xi\left(2^{k j}\right)}{2^{7 k j}} \\
& \left\|\psi(r)-N_{9}(r)\right\| \leq \frac{1}{512} \sum_{k=\frac{1-j}{2}}^{\infty} \frac{\xi\left(2^{k j}\right)}{2^{9 k j}}
\end{aligned}
$$

for all $p, q, r \in X$. The mapping $Q_{5}(p), S_{7}(q)$ and $N_{9}(r)$ is defined by

$$
\begin{aligned}
& Q_{5}(p)=\lim _{n \rightarrow \infty} \frac{\vartheta\left(2^{n j} p\right)}{2^{5 n j}} \\
& S_{7}(q)=\lim _{n \rightarrow \infty} \frac{\zeta\left(2^{n j} q\right)}{2^{7 n j}} \\
& N_{9}(r)=\lim _{n \rightarrow \infty} \frac{\varsigma\left(2^{n j} r\right)}{2^{9 n j}}
\end{aligned}
$$

for all $p, q, r \in X$.

Proof. Assume $j=1$. Using Oddness of $\vartheta, \eta$ and $\psi$ in (2.4), (2.5) and (2.6) we get

$$
\begin{aligned}
& \|\vartheta(2 p)-32 \vartheta(p)\| \leq \xi(p) \\
& \|\eta(2 q)-128 \eta(q)\| \leq \zeta(q) \\
& \|\psi(2 r)-512 \psi(r)\| \leq \varsigma(r)
\end{aligned}
$$

for all $p, q, r \in X$. It follows from above inequalities

$$
\begin{aligned}
& \left\|\vartheta(p)-\frac{\vartheta(2 p)}{2^{5}}\right\| \leq \frac{\xi(p)}{32} \\
& \left\|\eta(q)-\frac{\eta(2 q)}{2^{7}}\right\| \leq \frac{\zeta(q)}{128} \\
& \left\|\psi(r)-\frac{\psi(2 r)}{2^{9}}\right\| \leq \frac{\varsigma(r)}{512}
\end{aligned}
$$

for all $p, q, r \in X$. Now replacing $p$ by $2 p$ and dividing by 32 in (2.16), $q$ by $2 q$ and dividing by 128 in (2.17), $r$ by $2 r$ and dividing by 512 in (2.18) we get

$$
\begin{aligned}
& \left\|\frac{\vartheta(2 p)}{2^{5}}-\frac{\vartheta\left(2^{2} p\right)}{2^{10}}\right\| \leq \frac{\xi(2 p)}{2^{10}} \\
& \left\|\frac{\eta(2 q)}{2^{7}}-\frac{\eta\left(2^{2} q\right)}{2^{14}}\right\| \leq \frac{\zeta(2 q)}{2^{14}} \\
& \left\|\frac{\psi(2 r)}{2^{9}}-\frac{\psi\left(2^{2} r\right)}{2^{18}}\right\| \leq \frac{\varsigma(2 r)}{2^{18}}
\end{aligned}
$$


for all $p, q, r \in X$. With the help of triangle inequality from (2.16) and (2.19); (2.17) and (2.20); (2.18) and (2.21); we achieve the subsequent inequalities

$$
\begin{aligned}
& \left\|\vartheta(p)-\frac{\vartheta\left(2^{2} p\right)}{2^{10}}\right\| \\
& \leq\left\|\vartheta(p)-\frac{\vartheta(2 p)}{2^{5}}\right\|+\left\|\frac{\vartheta(2 p)}{2^{5}}-\frac{\vartheta\left(2^{2} p\right)}{2^{10}}\right\| \\
& \leq \frac{1}{2^{5}}\left[\xi(p)+\frac{\xi(2 p)}{2^{5}}\right] \\
& \left\|\eta(q)-\frac{\eta\left(2^{2} q\right)}{2^{14}}\right\| \\
& \quad \leq\left\|\eta(q)-\frac{\eta(2 q)}{2^{7}}\right\|+\left\|\frac{\eta(2 p)}{2^{7}}-\frac{\eta\left(2^{2} q\right)}{2^{14}}\right\| \\
& \quad \leq \frac{1}{2^{7}}\left[\zeta(q)+\frac{\zeta(2 q)}{2^{14}}\right] \\
& \left\|\psi(r)-\frac{\psi\left(2^{2} r\right)}{2^{18}}\right\| \\
& \quad \leq\left\|\psi(r)-\frac{\psi(2 r)}{2^{9}}\right\|+\left\|\frac{\psi(2 r)}{2^{9}}-\frac{\psi\left(2^{2} r\right)}{2^{18}}\right\| \\
& \quad \leq \frac{1}{2^{9}}\left[\varsigma(r)+\frac{\varsigma(2 r)}{2^{18}}\right]
\end{aligned}
$$

for all $p, q, r \in X$. In general for any positive integer $n$, we get

$$
\begin{aligned}
& \left\|\vartheta(p)-\frac{\vartheta\left(2^{n} p\right)}{2^{5 n}}\right\| \leq \frac{1}{32} \sum_{k=0}^{n-1} \frac{\xi\left(2^{k} p\right)}{2^{5 p}} \\
& \left\|\eta(q)-\frac{\eta\left(2^{n} q\right)}{2^{7 n}}\right\| \leq \frac{1}{128} \sum_{k=0}^{n-1} \frac{\zeta\left(2^{k} q\right)}{2^{7 q}} \\
& \left\|\psi(r)-\frac{\psi\left(2^{n} r\right)}{2^{9 n}}\right\| \leq \frac{1}{512} \sum_{k=0}^{n-1} \frac{\varsigma\left(2^{k} r\right)}{2^{9 r}}
\end{aligned}
$$

for all $p, q, r \in X$. In order to prove the convergence of the sequence

$$
\left\{\frac{\vartheta\left(2^{n} p\right)}{2^{5 n}}\right\},\left\{\frac{\eta\left(2^{n} q\right)}{2^{7 n}}\right\},\left\{\frac{\psi\left(2^{n} r\right)}{2^{9 n}}\right\},
$$

replace $p$ by $2^{m} p$ and dividing by $2^{5 m}$ in (2.25), $q$ by $2^{m} q$ and dividing by $2^{7 m}$ in (2.26), and $r$ by $2^{m} r$ and dividing by $2^{9 m}$ in (2.27), for any $m, n>0$, we deduce

$$
\begin{aligned}
\left\|\frac{\vartheta\left(2^{m} p\right)}{2^{5 m}}-\frac{\vartheta\left(2^{n+m} p\right)}{2^{5(n+m)}}\right\| & =\frac{1}{2^{5 m}}\left\|\vartheta\left(2^{m} p\right)-\frac{\vartheta\left(2^{n} \cdot 2^{m} p\right)}{2^{5 n}}\right\| \\
& \leq \frac{1}{32} \sum_{k=0}^{n-1} \frac{\xi\left(2^{k+m} p\right)}{2^{5(k+m)}} \\
& \leq \frac{1}{32} \sum_{k=0}^{\infty} \frac{\xi\left(2^{k+m}\right)}{2^{5(k+m)}} \\
& \rightarrow 0 \text { as } m \rightarrow \infty
\end{aligned}
$$

$$
\begin{aligned}
\left\|\frac{\eta\left(2^{m} q\right)}{2^{7 m}}-\frac{\eta\left(2^{n+m} q\right)}{2^{7(n+m)}}\right\| & =\frac{1}{2^{7 m}}\left\|\eta\left(2^{m} q\right)-\frac{\eta\left(2^{n} \cdot 2^{m} q\right)}{2^{7 n}}\right\| \\
& \leq \frac{1}{128} \sum_{k=0}^{n-1} \frac{\zeta\left(2^{k+m} q\right)}{2^{7(k+m)}} \\
& \leq \frac{1}{128} \sum_{k=0}^{\infty} \frac{\zeta\left(2^{k+m}\right)}{2^{7(k+m)}} \\
& \rightarrow 0 \text { as } m \rightarrow \infty \\
\left\|\frac{\psi\left(2^{m} r\right)}{2^{9 m}}-\frac{\psi\left(2^{n+m} r\right)}{2^{9(n+m)}}\right\| & =\frac{1}{2^{9 m}}\left\|\psi\left(2^{m} r\right)-\frac{\psi\left(2^{n} \cdot 2^{m} r\right)}{2^{9 n}}\right\| \\
& \leq \frac{1}{512} \sum_{k=0}^{n-1} \frac{\varsigma\left(2^{k+m} r\right)}{2^{9(k+m)}} \\
& \leq \frac{1}{512} \sum_{k=0}^{\infty} \frac{\varsigma\left(2^{k+m}\right)}{2^{9(k+m)}} \\
& \rightarrow 0 \text { as } m \rightarrow \infty
\end{aligned}
$$

for all $p, q, r \in X$. Hence the sequence

$$
\left\{\frac{\vartheta\left(2^{n} p\right)}{2^{5 n}}\right\},\left\{\frac{\eta\left(2^{n} q\right)}{2^{7 n}}\right\},\left\{\frac{\psi\left(2^{n} r\right)}{2^{9 n}}\right\}
$$

is Cauchy sequence. Since $Y$ is complete, there exists a mapping $Q_{5}, S_{7}, N_{9}: X \rightarrow Y$ such that

$$
\begin{aligned}
& Q_{5}(p)=\lim _{n \rightarrow \infty} \frac{\vartheta\left(2^{n} p\right)}{2^{5 n}} \forall p \in X . \\
& S_{7}(q)=\lim _{n \rightarrow \infty} \frac{\eta\left(2^{n} p\right)}{2^{7 n}} \forall q \in X . \\
& N_{9}(r)=\lim _{n \rightarrow \infty} \frac{\psi\left(2^{n} p\right)}{2^{9 n}} \forall r \in X .
\end{aligned}
$$

Letting $n \rightarrow \infty$ in (2.25), (2.26) and (2.27), we see that (2.7),(2.8) and (2.9) holds for all $p, q, r \in X$. To prove that $Q_{5}, S_{7}, N_{9}$ satisfies (1.3), (1.4), and (1.5), replace $p$ by $2^{n} p$ and dividing by $2^{5 n}$ in (2.4), $q$ by $2^{n} q$ and dividing by $2^{7 n}$ in (2.5), and $r$ by $2^{n} r$ and dividing by $2^{9 n}$ in (2.6), we obtain

$$
\begin{aligned}
& \frac{1}{2^{5 n}}\left\|\vartheta\left(2.2^{n} p\right)-48 \vartheta\left(2^{n} p\right)-16 \vartheta\left(-2^{n} p\right)\right\| \leq \frac{1}{2^{5 n}} \xi\left(2^{n} p\right) \\
& \frac{1}{2^{7 n}}\left\|\rho\left(2.2^{n} q\right)-192 \rho\left(2^{n} q\right)-64 \rho\left(-2^{n} q\right)\right\| \leq \frac{1}{2^{7 n}} \zeta\left(2^{n} q\right) \\
& \frac{1}{2^{9 n}}\left\|\psi\left(2.2^{n} r\right)-768 \psi\left(2^{n} r\right)-256 \psi\left(-2^{n} r\right)\right\| \leq \frac{1}{2^{9 n}} \varsigma\left(2^{n} r\right)
\end{aligned}
$$

for all $p, q, r \in X$. Letting $n \rightarrow \infty$ in the above inequality and using the definition of $Q_{5}(p), S_{7}(q)$ and $N_{9}(r)$, we see that

$$
\begin{aligned}
& Q_{5}(2 p)=48 Q_{5}(p)+16 Q_{5}(-p) \\
& S_{7}(2 q)=192 S_{7}(q)+64 S_{7}(-q) \\
& N_{9}(2 r)=768 N_{9}(r)+256 N_{9}(-r)
\end{aligned}
$$


Hence $Q_{5}(p), S_{7}(q)$ and $N_{9}(r)$ satisfies (1.3), (1.4), and (1.5) for all $p, q, r \in X$. To prove $Q_{5}(p), S_{7}(q)$ and $N_{9}(r)$ is unique, we let $\breve{Q}_{5}(p),{\breve{S_{7}}}_{7}(q)$ and $\breve{N}_{9}(r)$ be another mapping satisfying (1.3), (1.4), and (1.5) and (2.7),(2.8) and (2.9) then

$$
\begin{aligned}
& \left\|Q_{5}(p)-\check{Q}_{5}(p)\right\| \\
& =\frac{1}{2^{5 n}}\left\|Q_{5}\left(2^{n} p\right)-\check{Q}_{5}\left(2^{n} p\right)\right\| \\
& \leq \frac{1}{2^{5 n}}\left\{\left\|Q_{5}\left(2^{n} p\right)-\vartheta\left(2^{n} p\right)\right\|+\left\|\vartheta\left(2^{n} p\right)-\check{Q}_{5}\left(2^{n} p\right)\right\|\right\} \\
& \leq \sum_{k=0}^{\infty} \frac{2 \xi\left(2^{k+n} p\right)}{2^{5(k+n)}} \\
& \rightarrow 0 \text { as } n \rightarrow \infty \\
& \left\|S_{7}(q)-\check{S_{7}}(q)\right\| \\
& =\frac{1}{2^{7 n}}\left\|S_{7}\left(2^{n} q\right)-\breve{S}_{7}\left(2^{n} q\right)\right\| \\
& \leq \frac{1}{2^{7 n}}\left\{\left\|S_{7}\left(2^{n} q\right)-\eta\left(2^{n} q\right)\right\|+\left\|\eta\left(2^{n} p\right)-\check{S}_{7}\left(2^{n} q\right)\right\|\right\} \\
& \leq \sum_{k=0}^{\infty} \frac{2 \xi\left(2^{k+n} q\right)}{2^{7(k+n)}} \\
& \rightarrow 0 \text { as } n \rightarrow \infty \\
& \left\|N_{9}(r)-\check{N_{9}}(r)\right\| \\
& =\frac{1}{2^{9 n}}\left\|N_{9}\left(2^{n} r\right)-\check{N_{9}}\left(2^{n} r\right)\right\| \\
& \leq \frac{1}{2^{9 n}}\left\{\left\|N_{9}\left(2^{n} r\right)-\psi\left(2^{n} r\right)\right\|+\left\|\psi\left(2^{n} r\right)-\check{N}_{9}\left(2^{n} r\right)\right\|\right\} \\
& \leq \sum_{k=0}^{\infty} \frac{2 \varsigma\left(2^{k+n} r\right)}{2^{9(k+n)}} \\
& \rightarrow 0 \text { as } n \rightarrow \infty
\end{aligned}
$$

for all $p, q, r \in X$. Hence $Q_{5}, S_{7}$ and $N_{9}$ is unique.

For $j=-1$, we can prove a similar stability result. This completes the proof of the theorem.

The following Corollary is an immediate consequence of Theorem 2.1 concerning the Ulam-Hyers [16], Ulam-HyersRassias [27] and Ulam-JRassias [26] stabilities of (1.3), (1.4), and (1.5).

Corollary 2.2. Let $\lambda$ and $s$ be nonnegative real numbers. Let an odd function $\vartheta, \eta, \psi: X \rightarrow Y$ satisfies the inequality

$$
\begin{aligned}
& \|\vartheta(2 p)-48 \vartheta(p)-16 \vartheta(-p)\| \leq \begin{cases}\lambda, & \\
\lambda\|p\|^{s}, & s \neq 5 \\
(2.28)\end{cases}
\end{aligned}
$$

$$
\|\psi(2 r)-768 \psi(r)-256 \psi(-r)\| \leq\left\{\begin{array}{l}
\lambda, \\
\lambda\|r\|^{s}, \quad s \neq 9 ;
\end{array}\right.
$$

for all $p, q, r \in X$. Then there exists a unique quintic,septic,nonic mapping $Q_{5}, S_{7}, N_{9}: X \rightarrow Y$ such that

$$
\begin{aligned}
& \left\|\vartheta(p)-Q_{5}(p)\right\| \leq\left\{\begin{array}{l}
\frac{\lambda}{31}, \\
\frac{\lambda}{\left|2^{5}-2^{s}\right|},
\end{array}\right. \\
& \left\|\eta(p)-S_{7}(q)\right\| \leq\left\{\begin{array}{l}
\frac{\lambda}{127}, \\
\frac{\lambda|| p \|^{s}}{\left|2^{7}-2^{s}\right|}
\end{array}\right. \\
& \left\|\psi(r)-N_{9}(r)\right\| \leq\left\{\begin{array}{l}
\frac{\lambda}{511}, \\
\frac{\lambda|| p \|^{s}}{\left|2^{9}-2^{s}\right|},
\end{array}\right.
\end{aligned}
$$

for all $p, q, r \in X$.

\section{Stability Results : Even Case}

In this section, the generalized Ulam-Hyers stability of the functional equation (1.3), (1.4) and (1.5) even case is investigated.

Theorem 3.1. Let $j \in\{-1,1\}$ and $\xi, \zeta, \varsigma: X \rightarrow[0, \infty)$ be a function such that

$$
\lim _{n \rightarrow \infty} \frac{\xi\left(2^{n j} p\right)}{2^{6 n j}}=0
$$

$$
\begin{aligned}
& \lim _{n \rightarrow \infty} \frac{\zeta\left(2^{n j} q\right)}{2^{8 n j}}=0 \\
& \lim _{n \rightarrow \infty} \frac{\varsigma\left(2^{n j} r\right)}{2^{10 n j}}=0
\end{aligned}
$$

for all $p, q, r \in X$. Let $\vartheta, \eta, \psi: X \rightarrow Y$ be a even function satisfying the inequality

$$
\|\vartheta(2 p)-48 \vartheta(p)-16 \vartheta(-p)\| \leq \xi(p)
$$

$$
\|\eta(2 q)-192 \eta(q)-64 \eta(-q)\| \leq \zeta(q)
$$

$$
\|\psi(2 r)-768 \psi(r)-256 \psi(-r)\| \leq \varsigma(r)
$$


for all $p, q, r \in X$. Then there exists a unique sextic,octic,decic mapping $S_{6}, O_{8}, D_{10}: X \rightarrow Y$ and satisfying the functional equation (1.3), (1.4) and (1.5) such that

$$
\begin{aligned}
& \left\|\vartheta(p)-S_{6}(p)\right\| \leq \frac{1}{64} \sum_{k=\frac{1-j}{2}}^{\infty} \frac{\xi\left(2^{k j}\right)}{2^{6 k j}} \\
& \left\|\eta(q)-O_{8}(q)\right\| \leq \frac{1}{256} \sum_{k=\frac{1-j}{2}}^{\infty} \frac{\xi\left(2^{k j}\right)}{2^{8 k j}} \\
& \left\|\psi(r)-D_{10}(r)\right\| \leq \frac{1}{1024} \sum_{k=\frac{1-j}{2}}^{\infty} \frac{\xi\left(2^{k j}\right)}{2^{10 k j}}
\end{aligned}
$$

for all $p, q, r \in X$. The mapping $S_{6}(p), O_{8}(q)$ and $D_{10}(r)$ is defined by

$$
\begin{aligned}
& S_{6}(p)=\lim _{n \rightarrow \infty} \frac{\vartheta\left(2^{n j} x\right)}{2^{6 n j}} \\
& O_{8}(q)=\lim _{n \rightarrow \infty} \frac{\zeta\left(2^{n j} x\right)}{2^{8 n j}} \\
& D_{10}(r)=\lim _{n \rightarrow \infty} \frac{\varsigma\left(2^{n j} x\right)}{2^{10 n j}}
\end{aligned}
$$

for all $p, q, r \in X$.

Proof. Assume $j=1$. Using evenness of $\vartheta, \eta$ and $\psi$ in (3.4), (3.5) and (3.6) we get

$$
\begin{aligned}
& \|\vartheta(2 p)-64 \vartheta(p)\| \leq \xi(p) \\
& \|\eta(2 q)-256 \eta(q)\| \leq \zeta(q) \\
& \|\psi(2 r)-1024 \psi(r)\| \leq \varsigma(r)
\end{aligned}
$$

for all $p, q, r \in X$. The Rest of the Proof is similar to that of Theorem 2.1 .

Corollary 3.2. Let $\lambda$ and $s$ be nonnegative real numbers. Let an even function $\vartheta, \eta, \psi: X \rightarrow Y$ satisfies the inequality

$$
\begin{aligned}
& \|\vartheta(2 p)-48 \vartheta(p)-16 \vartheta(-p)\| \leq \begin{cases}\lambda, & \\
\lambda\|p\|^{s}, & s \neq 6 \\
(3.16)\end{cases}
\end{aligned}
$$

$$
\|\psi(2 r)-768 \psi(r)-256 \psi(-r)\| \leq\left\{\begin{array}{l}
\lambda, \\
\lambda\|r\|^{s}, \quad s \neq 10
\end{array}\right.
$$

for all $p, q, r \in X$. Then there exists a unique sextic,octic,decic mapping $S_{6}, O_{8}, D_{10}: X \rightarrow Y$ such that

$$
\begin{aligned}
& \left\|\vartheta(p)-S_{6}(p)\right\| \leq\left\{\begin{array}{l}
\frac{\lambda}{63}, \\
\frac{\left.\lambda|| p\right|^{s}}{\left|2^{6}-2^{s}\right|}
\end{array}\right. \\
& \left\|\eta(p)-O_{8}(q)\right\| \leq\left\{\begin{array}{l}
\frac{\lambda}{253}, \\
\frac{\lambda|| p||^{s}}{\left|2^{8}-2^{s}\right|}
\end{array}\right.
\end{aligned}
$$

$$
\left\|\psi(r)-D_{10}(r)\right\| \leq\left\{\begin{array}{l}
\frac{\lambda}{1023}, \\
\frac{\left.\lambda|| p\right|^{s}}{\left|2^{10}-2^{s}\right|}
\end{array}\right.
$$

for all $p, q, r \in X$

\section{Stability Results : Mixed Case}

In this section, the generalized Ulam-Hyers stability of the functional equation (1.3), (1.4) and (1.5) mixed case is proved.

Theorem 4.1. Let $j \in\{-1,1\}$ and $\xi, \zeta, \varsigma: X \rightarrow[0, \infty)$ be a function satisfying (2.1), (2.2) and (2.3) also (3.1), (3.2) and (3.3) for all $p, q, r \in X$. Let $\vartheta, \eta, \psi: X \rightarrow Y$ be a function satisfying the inequality

$$
\|\vartheta(2 p)-48 \vartheta(p)-16 \vartheta(-p)\| \leq \xi(p)
$$

$$
\|\eta(2 q)-192 \eta(q)-64 \eta(-q)\| \leq \zeta(q)
$$

$$
\|\psi(2 r)-768 \psi(r)-256 \psi(-r)\| \leq \varsigma(r)
$$

for all $p, q, r \in X$. Then there exists a unique quintic,septic,nonic mapping $Q_{5}, S_{7}, N_{9}: X \rightarrow Y$ and a unique sextic,octic,decic mapping $S_{6}, O_{8}, D_{10}: X \rightarrow Y$ such that

$$
\begin{aligned}
& \left\|\vartheta(p)-Q_{5}(p)-S_{6}(p)\right\| \\
& \leq \frac{1}{2}\left[\frac{1}{32} \sum_{k=\frac{1-j}{2}}^{\infty}\left(\frac{\xi\left(2^{k j} p\right)}{2^{5 k j}}+\frac{\xi\left(-2^{k j} p\right)}{2^{5 k j}}\right)\right. \\
& \left.\quad+\frac{1}{64} \sum_{k=\frac{1-j}{2}}^{\infty}\left(\frac{\xi\left(2^{k j} p\right)}{2^{6 k j}}+\frac{\xi\left(-2^{k j} p\right)}{2^{6 k j}}\right)\right]
\end{aligned}
$$




$$
\begin{aligned}
& \left\|\eta(q)-S_{7}(q)-O_{8}(q)\right\| \\
& \leq \frac{1}{2}\left[\frac{1}{128} \sum_{k=\frac{1-j}{2}}^{\infty}\left(\frac{\zeta\left(2^{k j} q\right)}{2^{7 k j}}+\frac{\zeta\left(-2^{k j} q\right)}{2^{7 k j}}\right)\right. \\
& \left.\quad+\frac{1}{256} \sum_{k=\frac{1-j}{2}}^{\infty}\left(\frac{\zeta\left(2^{k j} q\right)}{2^{8 k j}}+\frac{\zeta\left(-2^{k j} q\right)}{2^{8 k j}}\right)\right] \\
& \left\|\psi(r)-N_{9}(r)-D_{10}(r)\right\| \\
& \leq \frac{1}{2}\left[\frac { 1 } { 5 1 2 } \sum _ { k = \frac { 1 - j } { 2 } } ^ { \infty } \left(\frac{\varsigma\left(2^{k j} r\right)}{2^{2 k j}}+\frac{\varsigma\left(-2^{k j} r\right)}{\left.2^{9 k j}\right)}\right.\right. \\
& \left.\quad+\frac{1}{1024} \sum_{k=\frac{1-j}{2}}^{\infty}\left(\frac{\zeta\left(2^{k j} p\right)}{2^{10 k j}}+\frac{\varsigma\left(-2^{k j} r\right)}{2^{10 k j}}\right)\right]
\end{aligned}
$$

for all $p, q, r \in X$. The mapping $Q_{5}(p), S_{7}(q)$ and $N_{9}(r)$ also $S_{6}(p), O_{8}(q)$ and $D_{10}(r)$ are defined in (2.10), (2.11) and (2.12); (3.10), (3.11) and (3.11) respectively for all $p, q, r \in X$.

Proof. Let

$$
\begin{aligned}
& \vartheta_{o}(p)=\frac{\vartheta(p)-\vartheta(-p)}{2} \\
& \eta_{o}(q)=\frac{\eta(q)-\eta(-q)}{2} \\
& \psi_{o}(r)=\frac{\psi(r)-\psi(-r)}{2}
\end{aligned}
$$

for all $p, q, r \in X$. Then $\vartheta_{o}(0)=0, \eta_{o}(0)=0$ and $\psi_{o}(0)=0$ and $\vartheta_{o}(-p)=-\vartheta_{o}(p), \eta_{o}(-q)=-\eta_{o}(q), \psi_{o}(-r)=-\psi_{o}(r)$ for all $p, q, r \in X$. Hence

$$
\begin{aligned}
& \left\|\vartheta_{0}(2 p)-48 \vartheta_{o}(p)-16 \vartheta_{o}(-p)\right\| \leq \frac{\xi(p)}{2}+\frac{\xi(-p)}{2} \\
& \left\|\eta_{o}(2 q)-192 \eta_{o}(q)-64 \eta_{o}(-q)\right\| \leq \frac{\zeta(q)}{2}+\frac{\zeta(-q)}{2} \\
& \left\|\psi_{o}(2 r)-768 \psi_{o}(r)-256 \psi_{o}(-r)\right\| \leq \frac{\zeta(q)}{2}+\frac{\varsigma(-q)}{2}
\end{aligned}
$$

for all $p, q, r \in X$. By Theorem 2.1, we have

$$
\begin{aligned}
& \left\|\vartheta(p)-Q_{5}(p)\right\| \leq \frac{1}{32} \sum_{k=\frac{1-j}{2}}^{\infty}\left(\frac{\xi\left(2^{k j}\right)}{2^{5 k j}}+\frac{\xi\left(-2^{k j}\right)}{2^{5 k j}}\right) \\
& \left\|\eta(q)-S_{7}(q)\right\| \leq \frac{1}{128} \sum_{k=\frac{1-j}{2}}^{\infty}\left(\frac{\xi\left(2^{k j}\right)}{2^{7 k j}}+\frac{\xi\left(-2^{k j}\right)}{2^{7 k j}}\right)
\end{aligned}
$$

$$
\left\|\psi(r)-N_{9}(r)\right\| \leq \frac{1}{512} \sum_{k=\frac{1-j}{2}}^{\infty}\left(\frac{\xi\left(2^{k j}\right)}{2^{9 k j}}+\frac{\xi\left(-2^{k j}\right)}{2^{9 k j}}\right)
$$

for all $p, q, r \in X$. Also, Let

$$
\begin{gathered}
\vartheta_{e}(p)=\frac{\vartheta(p)+\vartheta(-p)}{2} \\
\eta_{e}(q)=\frac{\eta(q)+\eta(-q)}{2} \\
\psi_{e}(r)=\frac{\psi_{d}(r)+\psi_{d}(-r)}{2}
\end{gathered}
$$

for all $p, q, r \in X$. Then $\vartheta_{e}(0)=0, \eta_{e}(0)=0$ and $\psi_{e}(0)=0$ and $\vartheta_{e}(-p)=\vartheta_{e}(p), \eta_{e}(-q)=\eta_{e}(q), \psi_{e}(-r)=\psi_{e}(r)$ for all $p, q, r \in X$. Hence

$$
\left\|\vartheta_{e}(2 p)-48 \vartheta_{e}(p)-16 \vartheta_{e}(-p)\right\| \leq \frac{\xi(p)}{2}+\frac{\xi(-p)}{2}
$$

$$
\begin{gathered}
\left\|\eta_{e}(2 q)-192 \eta_{e}(q)-64 \eta_{e}(-q)\right\| \leq \frac{\zeta(q)}{2}+\frac{\zeta(-q)}{2} \\
\left\|\psi_{e}(2 r)-768 \psi_{e}(r)-256 \psi_{e}(-r)\right\| \leq \frac{\varsigma(r)}{2}+\frac{\varsigma(-r)}{2}
\end{gathered}
$$

for all $p, q, r \in X$. By Theorem 3.1, we have

$$
\begin{gathered}
\left\|\vartheta(p)-S_{6}(p)\right\| \leq \frac{1}{64} \sum_{k=\frac{1-j}{2}}^{\infty}\left(\frac{\xi\left(2^{k j}\right)}{2^{6 k j}}+\frac{\xi\left(-2^{k j}\right)}{2^{6 k j}}\right) \\
\left\|\eta(q)-O_{8}(q)\right\| \leq \frac{1}{256} \sum_{k=\frac{1-j}{2}}^{\infty}\left(\frac{\xi\left(2^{k j}\right)}{2^{8 k j}}+\frac{\xi\left(-2^{k j}\right)}{2^{8 k j}}\right) \\
\left\|\psi(r)-D_{10}(r)\right\| \leq \frac{1}{1024} \sum_{k=\frac{1-j}{2}}^{\infty}\left(\frac{\xi\left(2^{k j}\right)}{2^{10 k j}}+\frac{\xi\left(-2^{k j}\right)}{2^{10 k j}}\right)
\end{gathered}
$$

for all $p, q, r \in X$. Define

$$
\begin{aligned}
& \vartheta(p)=\vartheta_{e}(p)+\vartheta_{o}(p) \\
& \vartheta(q)=\vartheta_{e}(q)+\vartheta_{o}(q) \\
& \vartheta(r)=\vartheta_{e}(r)+\vartheta_{o}(r)
\end{aligned}
$$


for all $p, q, r \in X$. From (4.10), (4.11) and (4.12); (4.16), (4.17) and (4.18); (4.19) (4.20) and (4.21) we arrive

$$
\begin{aligned}
& \left\|\vartheta(p)-Q_{5}(p)-S_{6}(p)\right\| \\
& =\left\|\vartheta_{e}(p)+\vartheta_{o}(p)-Q_{5}(p)-S_{6}(p)\right\| \\
& \leq\left\|\vartheta_{o}(p)-Q_{5}(p)\right\|+\left\|\vartheta_{e}(p)-S_{6}(p)\right\| \\
& \leq \frac{1}{64} \sum_{k=\frac{1-j}{2}}^{\infty}\left(\frac{\xi\left(2^{k j} p\right)}{2^{5 k j}}+\frac{\xi\left(-2^{k j} p\right)}{2^{5 k j}}\right) \\
& +\frac{1}{128} \sum_{k=\frac{1-j}{2}}^{\infty}\left(\frac{\xi\left(2^{k j} p\right)}{2^{6 k j}}+\frac{\xi\left(-2^{k j} p\right)}{2^{6 k j}}\right) \\
& \left\|\eta(q)-S_{7}(q)-O_{8}(q)\right\| \\
& =\left\|\eta_{e}(q)+\eta_{o}(q)-S_{7}(q)-O_{8}(q)\right\| \\
& \leq\left\|\eta_{o}(q)-S_{7}(q)\right\|+\left\|\eta_{e}(q)-O_{8}(q)\right\| \\
& \leq \frac{1}{256} \sum_{k=\frac{1-j}{2}}^{\infty}\left(\frac{\zeta\left(2^{k j} q\right)}{2^{7 k j}}+\frac{\zeta\left(-2^{k j} q\right)}{2^{7 k j}}\right) \\
& +\frac{1}{1024} \sum_{k=\frac{1-j}{2}}^{\infty}\left(\frac{\zeta\left(2^{k j} q\right)}{2^{8 k j}}+\frac{\zeta\left(-2^{k j} q\right)}{2^{8 k j}}\right) \\
& \left\|\psi(r)-N_{9}(r)-D_{10}(r)\right\| \\
& =\left\|\psi_{e}(r)+\psi_{o}(r)-N_{9}(r)-D_{10}(r)\right\| \\
& \leq\left\|\psi_{o}(r)-N_{9}(r)\right\|+\left\|\psi_{e}(r)-D_{10}(r)\right\| \\
& \leq \frac{1}{1024} \sum_{k=\frac{1-j}{2}}^{\infty}\left(\frac{\varsigma\left(2^{k j} r\right)}{2^{9 k j}}+\frac{\varsigma\left(-2^{k j} r\right)}{2^{9 k j}}\right) \\
& +\frac{1}{2048} \sum_{k=\frac{1-j}{2}}^{\infty}\left(\frac{\varsigma\left(2^{k j} r\right)}{2^{10 k j}}+\frac{\varsigma\left(-2^{k j} r\right)}{2^{10 k j}}\right)
\end{aligned}
$$

for all $p, q, r \in X$. Hence the theorem is proved.

Using Corollaries 2.2 and 3.2, we have the following Corollary concerning the stability of (1.3), (1.4), and (1.5).

Corollary 4.2. Let $\lambda$ and $s$ be nonnegative real numbers. Let an even function $\vartheta, \eta, \psi: X \rightarrow Y$ satisfies the inequality

$$
\|\vartheta(2 p)-48 \vartheta(p)-16 \vartheta(-p)\| \leq\left\{\begin{array}{l}
\lambda, \\
\lambda\|p\|^{s}, \quad s \neq 5,6 ;
\end{array}\right.
$$

$$
\|\eta(2 q)-192 \eta(q)-64 \eta(-q)\| \leq\left\{\begin{array}{l}
\lambda, \\
\lambda\|q\|^{s}, \quad s \neq 7,8 ;
\end{array}\right.
$$

$$
\|\psi(2 r)-768 \psi(r)-256 \psi(-r)\| \leq\left\{\begin{array}{l}
\lambda, \\
\lambda\|r\|^{s}, \quad s \neq 9,10 ;
\end{array}\right.
$$

for all $p, q, r \in X$. Then there exists a unique quintic,septic,nonic mapping $Q_{5}, S_{7}, N_{9}: X \rightarrow Y$ and a unique sextic,octic,decic mapping $S_{6}, O_{8}, D_{10}: X \rightarrow Y$ such that

$$
\begin{aligned}
& \left\|\vartheta(p)-Q_{5}(p)-S_{6}(p)\right\| \leq\left\{\begin{array}{l}
\left(\frac{\lambda}{31}+\frac{\lambda}{63}\right), \\
\left.\frac{\lambda \|\left. p\right|^{s}}{\left|2^{5}-2^{s}\right|}+\frac{\lambda\|p\|^{s}}{\left|2^{6}-2^{s}\right|}\right),
\end{array}\right. \\
& \left\|\eta(q)-S_{7}(q)-O_{8}(q)\right\| \leq\left\{\begin{array}{l}
\left(\frac{\lambda}{127}+\frac{\lambda}{253}\right) \\
\frac{\lambda \| p||^{s}}{\left|2^{7}-2^{s}\right|}+\frac{\lambda\|p\|^{s}}{\left|2^{8}-2^{s}\right|}
\end{array}\right), \\
& \left\|\psi(r)-N_{9}(r)-D_{10}(r)\right\| \leq\left\{\begin{array}{l}
\left(\frac{\lambda}{511}+\frac{\lambda}{1023}\right), \\
\frac{\lambda\|r\|^{s}}{\left|2^{9}-2^{s}\right|}+\frac{\lambda \|\left. r\right|^{s}}{\left|2^{10}-2^{s}\right|}
\end{array}\right),
\end{aligned}
$$

for all $p, q, r \in X$.

Theorem 4.3. [20](The alternative of fixed point) Suppose that for a complete generalized metric space $(X, d)$ and a strictly contractive mapping $T: X \rightarrow X$ with Lipschitz constant $L$. Then, for each given element $x \in X$, either

$\left(B_{1}\right) \quad d\left(T^{n} x, T^{n+1} x\right)=\infty \quad \forall n \geq 0$, or

$\left(B_{2}\right)$ there exists a natural number $n_{0}$ such that:

(i) $d\left(T^{n} x, T^{n+1} x\right)<\infty$ for all $n \geq n_{0}$;

(ii) The sequence $\left(T^{n} x\right)$ is convergent to a fixed point $y^{*}$ of $T$

(iii) $y^{*}$ is the unique fixed point of $T$ in the set $Y=\{y \in X$ : $\left.d\left(T^{n_{0}} x, y\right)<\infty\right\}$;

(iv) $d\left(y^{*}, y\right) \leq \frac{1}{1-L} d(y, T y)$ for all $y \in Y$.

\section{Stability Results : Fixed Point Method: Odd Case}

In this section, the generalized Ulam-Hyers stability of the functional equation (1.3), (1.4) and (1.5) odd case is proved by using fixed point method.

Theorem 5.1. Let $\vartheta, \eta, \psi: X \rightarrow Y$ be a mapping for which there exist a function $\xi, \zeta, \varsigma: X \rightarrow[0, \infty)$ with the condition

$$
\begin{aligned}
& \lim _{k \rightarrow \infty} \frac{\xi\left(\mu_{i}^{k} p\right)}{\mu_{i}^{5 k}}=0 \\
& \lim _{k \rightarrow \infty} \frac{\zeta\left(\mu_{i}^{k} q\right)}{\mu_{i}^{7 k}}=0
\end{aligned}
$$




$$
\lim _{k \rightarrow \infty} \frac{\varsigma\left(\mu_{i}^{k} r\right)}{\mu_{i}^{9 k}}=0
$$

where $\mu_{i}=2$ if $i=0$ and $\mu_{i}=\frac{1}{2}$ if $i=1$ such that the functional inequality with

$$
\begin{gathered}
\|\vartheta(2 p)-48 \vartheta(p)-16 \vartheta(-p)\| \leq \xi(p) \\
\|\eta(2 q)-192 \eta(q)-64 \eta(-q)\| \leq \zeta(q) \\
\|\psi(2 r)-768 \psi(r)-256 \psi(-r)\| \leq \varsigma(r)
\end{gathered}
$$

for all $p, q, r \in X$. If there exists $L=L(i)<1$ such that the function

$$
\begin{aligned}
& p \rightarrow \beta(p)=\xi\left(\frac{p}{2}\right), \\
& q \rightarrow \beta(q)=\zeta\left(\frac{q}{2}\right), \\
& r \rightarrow \beta(r)=\varsigma\left(\frac{r}{2}\right),
\end{aligned}
$$

has the property

$$
\begin{aligned}
& L \beta(p) \leq \frac{1}{\mu_{i}^{5}} \beta\left(p \mu_{i}\right) \\
& L \beta(q) \leq \frac{1}{\mu_{i}^{7}} \beta\left(q \mu_{i}\right) \\
& L \beta(r) \leq \frac{1}{\mu_{i}^{9}} \beta\left(r \mu_{i}\right)
\end{aligned}
$$

for all $p, q, r \in X$. Then there exists a unique quintic,septic,nonic mapping $Q_{5}, S_{7}, N_{9}: X \rightarrow Y$ satisfying the functional equation (1.3), (1.4) and (1.5) such that

$$
\begin{gathered}
\left\|\vartheta(p)-Q_{5}(p)\right\| \leq \frac{L^{1-i}}{1-L} \beta(p) \\
\left\|\eta(q)-S_{7}(q)\right\| \leq \frac{L^{1-i}}{1-L} \beta(q) \\
\left\|\psi(r)-N_{9}(r)\right\| \leq \frac{L^{1-i}}{1-L} \beta(r)
\end{gathered}
$$

holds for all $p, q, r \in X$.
Proof. Consider the set $\Psi=\{f / g: X \rightarrow Y, f(0)=0\}$ and introduce the generalized metric on $\Psi$,

$d(f, g)=\inf \{K \in(0, \infty):\|f(p)-g(p)\| \leq K \beta(p), p \in X\}$.

$d(f, g)=\inf \{K \in(0, \infty):\|f(q)-g(q)\| \leq K \beta(q), q \in X\}$.

$d(f, g)=\inf \{K \in(0, \infty):\|f(r)-g(r)\| \leq K \beta(r), r \in X\}$.

It is easy to see that $(\Psi, d)$ is complete. Define $T: \Psi \rightarrow \Psi$ by

$$
\begin{aligned}
& T f(p)=\frac{1}{\mu_{i}^{5}} f\left(\mu_{i} p\right) \\
& T f(q)=\frac{1}{\mu_{i}^{7}} f\left(\mu_{i} q\right) \\
& T f(r)=\frac{1}{\mu_{i}^{9}} f\left(\mu_{i} r\right)
\end{aligned}
$$

for all $p, q, r \in \Psi$. Now $f, g \in \Psi$,

$$
\begin{aligned}
& d(f, g) \leq K \\
\Rightarrow & \|f(p)-g(p)\| \leq K \beta(p), p \in X . \\
\Rightarrow & \left\|\frac{1}{\mu_{i}^{5}} f\left(\mu_{i} p\right)-\frac{1}{\mu_{i}^{5}} g\left(\mu_{i} p\right)\right\| \leq \frac{1}{\mu_{i}^{5}} K \beta\left(\mu_{i} p\right), p \in X, \\
\Rightarrow & \left\|\frac{1}{\mu_{i}^{5}} f\left(\mu_{i} p\right)-\frac{1}{\mu_{i}^{5}} g\left(\mu_{i} p\right)\right\| \leq L K \beta(p), p \in X, \\
\Rightarrow & \|T f(p)-T g(p)\| \leq L K \beta(p), p \in X, \\
\Rightarrow & d(T f, T g) \leq L K . \\
& d(f, g) \leq K \\
\Rightarrow & \|f(q)-g(q)\| \leq K \beta(q), q \in X . \\
\Rightarrow & \left\|\frac{1}{\mu_{i}^{7}} f\left(\mu_{i} q\right)-\frac{1}{\mu_{i}} g\left(\mu_{i} q\right)\right\| \leq \frac{1}{\mu_{i}^{7}} K \beta\left(\mu_{i} q\right), q \in X, \\
\Rightarrow & \left\|\frac{1}{\mu_{i}^{7}} f\left(\mu_{i} q\right)-\frac{1}{\mu_{i}^{7}} g\left(\mu_{i} q\right)\right\| \leq L K \beta(q), q \in X, \\
\Rightarrow & \|T f(q)-T g(q)\| \leq L K \beta(x), q \in X, \\
\Rightarrow & d(T f, T g) \leq L K . \\
& d(f, g) \leq K \\
\Rightarrow & \|f(r)-g(r)\| \leq K \beta(r), r \in X . \\
\Rightarrow & \left\|\frac{1}{\mu_{i}^{9}} f\left(\mu_{i} r\right)-\frac{1}{\mu_{i}^{9}} g\left(\mu_{i} r\right)\right\| \leq \frac{1}{\mu_{i}^{9}} K \beta\left(\mu_{i} r\right), r \in X, \\
\Rightarrow & \left\|\frac{1}{\mu_{i}^{9}} f\left(\mu_{i} r\right)-\frac{1}{\mu_{i}^{9}} g\left(\mu_{i} r\right)\right\| \leq L K \beta(r), r \in X, \\
\Rightarrow & \|T f(r)-T g(r)\| \leq L K \beta(r), r \in X, \\
\Rightarrow & d(T f, T g) \leq L K .
\end{aligned}
$$

This implies $d(T f, T g) \leq L d(f, g)$, for all $f, g \in \Psi$. i.e., $T$ is a strictly contractive mapping on $\Psi$ with Lipschitz constant $L$. Using oddness of $\vartheta, \eta$ and $\psi$ in (5.4), (5.5) and (5.6) we get

$$
\left\|\vartheta(p)-\frac{\vartheta(2 p)}{2^{5}}\right\| \leq \frac{\xi(p)}{32}
$$




$$
\begin{gathered}
\left\|\eta(q)-\frac{\eta(2 q)}{2^{7}}\right\| \leq \frac{\zeta(q)}{128} \\
\left\|\psi(r)-\frac{\psi(2 r)}{2^{9}}\right\| \leq \frac{\varsigma(r)}{512}
\end{gathered}
$$

for all $p, q, r \in X$. for all $p, q, r \in X$. Using (5.7),(5.8) and (5.9) for the case $i=0$ it reduces to

$$
\begin{aligned}
\left\|\vartheta(p)-\frac{\vartheta(2 p)}{2^{5}}\right\| & \leq \frac{1}{2} \beta(p) \\
\left\|\eta(q)-\frac{\eta(2 q)}{2^{7}}\right\| & \leq \frac{1}{2} \beta(q) \\
\left\|\psi(r)-\frac{\psi(2 r)}{2^{9}}\right\| & \leq \frac{1}{2} \beta(r)
\end{aligned}
$$

for all $p, q, r \in X$.

i.e., $\quad d(\vartheta, T \vartheta) \leq \frac{1}{2} \Rightarrow d(\vartheta, T \vartheta) \leq \frac{1}{2}=L=L^{1}<\infty$.

i.e., $\quad d(\eta, T \eta) \leq \frac{1}{2} \Rightarrow d(\eta, T \eta) \leq \frac{1}{2}=L=L^{1}<\infty$.

i.e., $\quad d(\psi, T \psi) \leq \frac{1}{2} \Rightarrow d(\psi, T \psi) \leq \frac{1}{2}=L=L^{1}<\infty$.

Again replacing $p=\frac{p}{2}$ in (5.13), $q=\frac{q}{2}$ in (5.14) and $r=\frac{r}{2}$ in (5.15) we get

$$
\begin{gathered}
\left\|\vartheta(p)-2^{5} \vartheta\left(\frac{p}{2}\right)\right\| \leq \xi\left(\frac{p}{2}\right) . \\
\left\|\eta(q)-2^{7} \eta\left(\frac{q}{2}\right)\right\| \leq \zeta\left(\frac{q}{2}\right) . \\
\left\|\psi(r)-2^{9} \psi\left(\frac{r}{2}\right)\right\| \leq \varsigma\left(\frac{r}{2}\right) .
\end{gathered}
$$

for all $p, q, r \in X$. Using (5.7),(5.8) and (5.9) for the case $i=1$ it reduces to

$$
\begin{aligned}
\left\|\vartheta(p)-2^{5} \vartheta\left(\frac{p}{2}\right)\right\| & \leq \beta(p) \\
\left\|\eta(q)-2^{7} \eta\left(\frac{q}{2}\right)\right\| & \leq \beta(q) \\
\left\|\psi(r)-2^{9} \psi\left(\frac{r}{2}\right)\right\| & \leq \beta(r)
\end{aligned}
$$

for all $p, q, r \in X$.

$$
\begin{array}{ll}
\text { i.e., } & d(\vartheta, T \vartheta) \leq 1 \Rightarrow d\left(\vartheta, T \vartheta \leq 1=L^{0}<\infty .\right. \\
\text { i.e., } & d(\eta, T \eta) \leq 1 \Rightarrow d(\eta, T \eta) \leq 1=L^{0}<\infty . \\
\text { i.e., } & d(\psi, T \psi) \leq 1 \Rightarrow d(\psi, T \psi) \leq 1=L^{0}<\infty .
\end{array}
$$

In above cases, we arrive

$$
d(\vartheta, T \vartheta) \leq L^{1-i}
$$

$$
\begin{aligned}
& d(\eta, T \eta) \leq L^{1-i} \\
& d(\psi, T \psi) \leq L^{1-i}
\end{aligned}
$$

Therefore $\left(B_{2}(i)\right)$ holds.

By $\left(B_{2}(i i)\right)$, it follows that there exists a fixed point $Q_{5}$, $S_{7}$ and $N_{9}$ of $T$ in $X$ such that

$$
Q_{5}(p)=\lim _{k \rightarrow \infty} \frac{\vartheta\left(\mu_{i}^{k} p\right)}{\mu_{i}^{k}} \quad \forall p \in X .
$$

$$
\begin{aligned}
& S_{7}(q)=\lim _{k \rightarrow \infty} \frac{\eta(q)\left(\mu_{i}^{k} q\right)}{\mu_{i}^{k}} \quad \forall q \in X . \\
& N_{9}(r)=\lim _{k \rightarrow \infty} \frac{\psi\left(\mu_{i}^{k} r\right)}{\mu_{i}^{k}} \quad \forall r \in X .
\end{aligned}
$$

In order to prove $Q_{5}, S_{7}, N_{9}: V \rightarrow B$ is quintic, septic, nonic. Replacing $p$ by $\left(\mu_{i}^{k} p\right), q$ by $\left(\mu_{i}^{k} q\right)$ and $r$ by $\left(\mu_{i}^{k} r\right)$ in (5.4), (5.5) and (5.6) and dividing by $\mu_{i}^{k}$, it follows from (5.1), (5.2) and (5.3); and (5.16),(5.17) and (5.18) $Q_{5}, S_{7}$ and $N_{9}$ satisfies (1.3), (1.4) and (1.5) for all $p, q, r \in X$. i.e., $Q_{5}, S_{7}$ and $N_{9}$ satisfies the functional equation (1.3), (1.4) and (1.5).

By $\left(B_{2}(i i i)\right), Q_{5}, S_{7}$ and $N_{9}$ is the unique fixed point of $T$ in the set

$$
\begin{gathered}
Y=\left\{Q_{5}(p) \in X: d\left(T \vartheta(p), Q_{5}(p)\right)<\infty\right\}, \\
Y=\left\{S_{7}(q) \in X: d\left(T \eta(q), S_{7}(q)\right)<\infty\right\}, \\
Y=\left\{N_{9}(r) \in X: d\left(T \psi(r), N_{9}(r)\right)<\infty\right\},
\end{gathered}
$$

using the fixed point alternative result $Q_{5}, S_{7}$ and $N_{9}$ is the unique function such that

$$
\begin{gathered}
\left\|\vartheta(p)-Q_{5}(p)\right\| \leq K \beta(p) \\
\left\|\eta(q)-S_{7}(q)\right\| \leq K \beta(q) \\
\left\|\psi(r)-N_{9}(r)\right\| \leq K \beta(r)
\end{gathered}
$$

for all $p, q, r \in X$ and $K>0$. Finally by $\left(B_{2}(i v)\right)$, we obtain

$$
\begin{aligned}
& d\left(\vartheta, Q_{5}\right) \leq \frac{1}{1-L} d(\vartheta, T \vartheta) \\
& d\left(\eta, S_{7}\right) \leq \frac{1}{1-L} d(\eta, T \eta) \\
& d\left(\psi, N_{9}\right) \leq \frac{1}{1-L} d(\psi, T \psi)
\end{aligned}
$$

this implies

$$
\begin{aligned}
d\left(\vartheta, Q_{5}\right) & \leq \frac{L^{1-i}}{1-L} \\
d\left(\eta, S_{7}\right) & \leq \frac{L^{1-i}}{1-L} \\
d\left(\psi, N_{9}\right) & \leq \frac{L^{1-i}}{1-L}
\end{aligned}
$$


Hence we conclude that

$$
\begin{gathered}
\left\|\vartheta(p)-Q_{5}(p)\right\| \leq \frac{L^{1-i}}{1-L} \beta(p) \\
\left\|\eta(q)-S_{7}(q)\right\| \leq \frac{L^{1-i}}{1-L} \beta(q), \\
\left\|\psi(r)-N_{9}(r)\right\| \leq \frac{L^{1-i}}{1-L} \beta(r) .
\end{gathered}
$$

for all $p, q, r \in X$. This completes the proof of the theorem.

Corollary 5.2. Let $\lambda$ and $s$ be nonnegative real numbers. Let an odd function $\vartheta, \eta, \psi: X \rightarrow Y$ satisfies the inequality

$$
\begin{aligned}
& \|\vartheta(2 p)-48 \vartheta(p)-16 \vartheta(-p)\| \leq \begin{cases}\lambda, \\
\lambda\|p\|^{s}, & s \neq 5 \\
(5.19)\end{cases} \\
& \|\eta(2 q)-192 \eta(q)-64 \eta(-q)\| \leq \begin{cases}\lambda, & \\
\lambda\|q\|^{s}, & s \neq 7 ;\end{cases} \\
& \|\psi(2 r)-768 \psi(r)-256 \psi(-r)\| \leq \begin{cases}\lambda, \\
\lambda\|r\|^{s}, & s \neq 9 ;\end{cases}
\end{aligned}
$$

for all $p, q, r \in X$. Then there exists a unique quintic,septic,nonic mapping $Q_{5}, S_{7}, N_{9}: X \rightarrow Y$ such that

$$
\begin{aligned}
& \left\|\vartheta(p)-Q_{5}(p)\right\| \leq\left\{\begin{array}{l}
\frac{\lambda}{31}, \\
\frac{\left.\lambda|| p\right|^{s}}{\left|2^{5}-2^{s}\right|}
\end{array}\right. \\
& \left\|\eta(p)-S_{7}(q)\right\| \leq\left\{\begin{array}{l}
\frac{\lambda}{127}, \\
\frac{\left.\lambda|| p\right|^{s}}{\left|2^{7}-2^{s}\right|},
\end{array}\right. \\
& \left\|\psi(r)-N_{9}(r)\right\| \leq\left\{\begin{array}{l}
\frac{\lambda}{511}, \\
\frac{\lambda|| p \|^{s}}{\left|2^{9}-2^{s}\right|},
\end{array}\right.
\end{aligned}
$$

for all $p, q, r \in X$.

Proof. Setting

$$
\begin{aligned}
& \xi(x)=\left\{\begin{array}{l}
\lambda \\
\lambda\left\{\|x\|^{s}\right\},
\end{array}\right. \\
& \zeta(x)=\left\{\begin{array}{l}
\lambda \\
\lambda\left\{\|x\|^{s}\right\},
\end{array}\right.
\end{aligned}
$$

$$
\varsigma(x)=\left\{\begin{array}{l}
\lambda \\
\lambda\left\{\|x\|^{s}\right\}
\end{array}\right.
$$

for all $p, q, r \in X$. Now

$$
\begin{aligned}
\frac{\xi\left(\mu_{i}^{k} p\right)}{\mu_{i}^{5 k}} & =\left\{\begin{array}{l}
\frac{\lambda}{\mu_{i}^{5 k}} \\
\frac{\lambda}{\mu_{i}^{5 k}}\left\{\left\|\mu_{i}^{k} p\right\|^{s}\right\}
\end{array}\right. \\
& =\left\{\begin{array}{l}
\rightarrow 0 \text { as } k \rightarrow \infty, \\
\rightarrow 0 \text { as } k \rightarrow \infty
\end{array}\right.
\end{aligned}
$$

$$
\begin{aligned}
& \frac{\zeta\left(\mu_{i}^{k} q\right)}{\mu_{i}^{7 k}}=\left\{\begin{array}{l}
\frac{\lambda}{\mu_{i}^{7 k}} \\
\frac{\lambda}{\mu_{i}^{7 k}}\left\{\left\|\mu_{i}^{k} q\right\|^{s}\right\}
\end{array}\right. \\
& =\left\{\begin{array}{l}
\rightarrow 0 \text { as } k \rightarrow \infty, \\
\rightarrow 0 \text { as } k \rightarrow \infty
\end{array}\right.
\end{aligned}
$$

$$
\begin{aligned}
\frac{\varsigma\left(\mu_{i}^{k} r\right)}{\mu_{i}^{9 k}} & =\left\{\begin{array}{l}
\frac{\lambda}{\mu_{i}^{9 k}} \\
\frac{\lambda}{\mu_{i}^{9 k}}\left\{\left\|\mu_{i}^{k} r\right\|^{s}\right\}
\end{array}\right. \\
& = \begin{cases}\rightarrow 0 \text { as } & k \rightarrow \infty \\
\rightarrow 0 \text { as } & k \rightarrow \infty\end{cases}
\end{aligned}
$$

i.e., (5.1),(5.2) and (5.3) is holds. But we have $\beta(p)=\xi\left(\frac{p}{2}\right)$, $\beta(q)=\zeta\left(\frac{q}{2}\right)$ and $\beta(r)=\varsigma\left(\frac{r}{2}\right)$. has the property

$$
\begin{aligned}
& L \beta(p) \leq \frac{1}{\mu_{i}^{5}} \beta\left(p \mu_{i}\right) \\
& L \beta(q) \leq \frac{1}{\mu_{i}^{7}} \beta\left(q \mu_{i}\right) \\
& L \beta(r) \leq \frac{1}{\mu_{i}^{9}} \beta\left(r \mu_{i}\right)
\end{aligned}
$$

for all $p, q, r \in V$. Hence

$$
\beta(p)=\xi\left(\frac{p}{2}\right)=\left\{\begin{array}{l}
\lambda \\
\frac{\lambda}{2^{s}}\|p\|^{s},
\end{array}\right.
$$

$$
\beta(q)=\zeta\left(\frac{q}{2}\right)=\left\{\begin{array}{l}
\lambda \\
\frac{\lambda}{2^{s}}\|q\|^{s},
\end{array}\right.
$$

$$
\beta(r)=\varsigma\left(\frac{r}{2}\right)=\left\{\begin{array}{l}
\lambda \\
\frac{\lambda}{2^{s}}\|r\|^{s},
\end{array}\right.
$$


Also,

$$
\begin{aligned}
\frac{1}{\mu_{i}^{5}} \beta\left(\mu_{i} p\right) & =\left\{\begin{array}{l}
\frac{\lambda}{\mu_{i}^{5}} \\
\frac{\lambda}{\mu_{i}^{5}}\left\|\mu_{i} p\right\|^{s}
\end{array}\right. \\
& =\left\{\begin{array}{l}
\mu_{i}^{-5} \beta(p) \\
\mu_{i}^{s-5} \beta(p)
\end{array}\right. \\
\frac{1}{\mu_{i}^{7}} \beta\left(\mu_{i} p\right) & =\left\{\begin{array}{l}
\frac{\lambda}{\mu_{i}^{7}} \\
\frac{\lambda}{\mu_{i}^{7}}\left\|\mu_{i} q\right\|^{s}
\end{array}\right. \\
& =\left\{\begin{array}{l}
\mu_{i}^{-7} \beta(q) \\
\mu_{i}^{s-7} \beta(q)
\end{array}\right. \\
\frac{1}{\mu_{i}^{9}} \beta\left(\mu_{i} p\right) & =\left\{\begin{array}{l}
\frac{\lambda}{\mu_{i}^{9}} \\
\frac{\lambda}{\mu_{i}^{9}}\left\|\mu_{i} r\right\|^{s}
\end{array}\right. \\
& =\left\{\begin{array}{l}
\mu_{i}^{-9} \beta(r) \\
\mu_{i}^{s-9} \beta(r)
\end{array}\right.
\end{aligned}
$$

Now from (5.10), (5.11) and (5.12), we prove the following cases

Case: $1 L=2^{-5}$, if $i=0$

$$
\left\|\vartheta(p)-Q_{5}(p)\right\| \leq \frac{L^{1-i}}{1-L} \beta(p)=\frac{\left(2^{-5}\right)^{1-0}}{1-(2)^{-5}} \lambda=\frac{\lambda}{31} .
$$

Case: $2 L=\frac{1}{2^{-5}}$, if $i=1$

$$
\left\|\vartheta(p)-Q_{5}(p)\right\| \leq \frac{L^{1-i}}{1-L} \beta(p)=\frac{\left(\frac{1}{2^{-5}}\right)^{1-1}}{1-\frac{1}{2^{-5}}} \lambda=\frac{\lambda}{-31} .
$$

Case: $1 L=2^{-7}$, if $i=0$

$$
\left\|\eta(q)-S_{7}(q)\right\| \leq \frac{L^{1-i}}{1-L} \beta(q)=\frac{\left(2^{-7}\right)^{1-0}}{1-(2)^{-7}} \lambda=\frac{\lambda}{127} .
$$

Case:2 $L=\frac{1}{2^{-7}}$, if $i=1$

$$
\left\|\eta(q)-S_{7}(q)\right\| \leq \frac{L^{1-i}}{1-L} \beta(q)=\frac{\left(\frac{1}{2^{-7}}\right)^{1-1}}{1-\frac{1}{2^{-7}}} \lambda=\frac{\lambda}{-127} .
$$

Case: $1 L=2^{-9}$, if $i=0$

$$
\left\|\psi(p)-N_{9}(r)\right\| \leq \frac{L^{1-i}}{1-L} \beta(r)=\frac{\left(2^{-9}\right)^{1-0}}{1-(2)^{-9}} \lambda=\frac{\lambda}{511} .
$$

Case:2 $L=\frac{1}{2^{-9}}$, if $i=1$

$$
\left\|\psi(p)-N_{9}(r)\right\| \leq \frac{L^{1-i}}{1-L} \beta(r)=\frac{\left(\frac{1}{2^{-9}}\right)^{1-1}}{1-\frac{1}{2^{-9}}} \lambda=\frac{\lambda}{-511} .
$$

Case: $1 L=2^{s-5}$, if $i=0$

$$
\begin{aligned}
\left\|\vartheta(p)-Q_{5}(p)\right\| & \leq \frac{L^{1-i}}{1-L} \beta(p) \\
& =\frac{\left(2^{s-5}\right)^{1-0}}{1-(2)^{s-5}} \frac{\lambda}{2^{s}}\|p\|^{s} \\
& =\frac{\lambda\|p\|^{s}}{2^{5}-2^{s}} .
\end{aligned}
$$

Case:2 $L=\frac{1}{2^{s-5}}$, if $i=1$

$$
\begin{aligned}
\left\|\vartheta(p)-Q_{5}(p)\right\| & \leq \frac{L^{1-i}}{1-L} \beta(p) \\
& =\frac{\left(\frac{1}{2^{s-5}}\right)^{1-1}}{1-\frac{1}{2^{s-5}}} \frac{\lambda}{2^{s}}\|p\|^{s} \\
& =\frac{\lambda\|p\|^{s}}{2^{s}-2^{5}} .
\end{aligned}
$$

Case: $1 L=2^{s-7}$, if $i=0$

$$
\begin{aligned}
\left\|\eta(q)-S_{7}(q)\right\| & \leq \frac{L^{1-i}}{1-L} \beta(q) \\
& =\frac{\left(2^{s-7}\right)^{1-0}}{1-(2)^{s-7}} \frac{\lambda}{2^{s}}\|q\|^{s} \\
& =\frac{\lambda\|q\|^{s}}{2^{7}-2^{s}} .
\end{aligned}
$$

Case:2 $L=\frac{1}{2^{s-7}}$, if $i=1$

$$
\begin{aligned}
\left\|\eta(q)-S_{7}(q)\right\| & \leq \frac{L^{1-i}}{1-L} \beta(q) \\
& =\frac{\left(\frac{1}{2^{s-7}}\right)^{1-1}}{1-\frac{1}{2^{s-7}}} \frac{\lambda}{2^{s}}\|q\|^{s} \\
& =\frac{\lambda\|q\|^{s}}{2^{s}-2^{7}} .
\end{aligned}
$$

Case: $1 L=2^{s-9}$, if $i=0$

$$
\begin{aligned}
\left\|\psi(p)-N_{9}(r)\right\| & \leq \frac{L^{1-i}}{1-L} \beta(r) \\
& =\frac{\left(2^{s-9}\right)^{1-0}}{1-(2)^{s-9}} \frac{\lambda}{2^{s}}\|r\|^{s} \\
& =\frac{\lambda\|r\|^{s}}{2^{9}-2^{s}}
\end{aligned}
$$

Case: $2 L=\frac{1}{2^{s-9}}$, if $i=1$

$$
\begin{aligned}
\left\|\psi(p)-N_{9}(r)\right\| & \leq \frac{L^{1-i}}{1-L} \beta(r) \\
& =\frac{\left(\frac{1}{2^{s-9}}\right)^{1-1}}{1-\frac{1}{2^{s-9}}} \frac{\lambda}{2^{s}}\|r\|^{s} \\
& =\frac{\lambda\|r\|^{s}}{2^{s}-2^{9}} .
\end{aligned}
$$


Hence the proof of the corollary.

\section{Stability Results: Even Case}

In this section, the generalized Ulam-Hyers stability of the functional equation (1.3), (1.4) and (1.5) even case is proved by using fixed point method. The proofs of the following Theorem and Corollary is similar tracing to that of Theorem 5.1 and Corollary 5.2. Hence we omit the proofs.

Theorem 6.1. Let $\vartheta, \eta, \psi: X \rightarrow Y$ be a mapping for which there exist a function $\xi, \zeta, \varsigma: X \rightarrow[0, \infty)$ with the condition

$$
\begin{aligned}
& \lim _{k \rightarrow \infty} \frac{\xi\left(\mu_{i}^{k} p\right)}{\mu_{i}^{6 k}}=0 \\
& \lim _{k \rightarrow \infty} \frac{\zeta\left(\mu_{i}^{k} q\right)}{\mu_{i}^{8 k}}=0 \\
& \lim _{k \rightarrow \infty} \frac{\varsigma\left(\mu_{i}^{k} r\right)}{\mu_{i}^{10 k}}=0
\end{aligned}
$$

where $\mu_{i}=2$ if $i=0$ and $\mu_{i}=\frac{1}{2}$ if $i=1$ such that the functional inequality with

$$
\begin{aligned}
& \|\vartheta(2 p)-48 \vartheta(p)-16 \vartheta(-p)\| \leq \xi(p) \\
& \|\eta(2 q)-192 \eta(q)-64 \eta(-q)\| \leq \zeta(q) \\
& \|\psi(2 r)-768 \psi(r)-256 \psi(-r)\| \leq \varsigma(r)
\end{aligned}
$$

for all $p, q, r \in X$. If there exists $L=L(i)<1$ such that the function

$$
\begin{aligned}
& p \rightarrow \beta(p)=\xi\left(\frac{p}{2}\right), \\
& q \rightarrow \beta(q)=\zeta\left(\frac{q}{2}\right), \\
& r \rightarrow \beta(r)=\varsigma\left(\frac{r}{2}\right),
\end{aligned}
$$

has the property

$$
\begin{aligned}
& L \beta(p) \leq \frac{1}{\mu_{i}^{6}} \beta\left(p \mu_{i}\right) \\
& L \beta(q) \leq \frac{1}{\mu_{i}^{8}} \beta\left(q \mu_{i}\right) \\
& L \beta(r) \leq \frac{1}{\mu_{i}^{10}} \beta\left(r \mu_{i}\right)
\end{aligned}
$$

for all $p, q, r \in X$. Then there exists a unique sextic,octic, decic mapping $S_{6}, O_{8}, D_{10}: X \rightarrow Y$ satisfying the functional equation (1.3), (1.4) and (1.5) such that

$$
\begin{gathered}
\left\|\vartheta(p)-S_{6}(p)\right\| \leq \frac{L^{1-i}}{1-L} \beta(p) \\
\left\|\eta(q)-O_{8}(q)\right\| \leq \frac{L^{1-i}}{1-L} \beta(q)
\end{gathered}
$$

$$
\left\|\psi(r)-D_{10}(r)\right\| \leq \frac{L^{1-i}}{1-L} \beta(r)
$$

holds for all $p, q, r \in X$.

Corollary 6.2. Let $\lambda$ and $s$ be nonnegative real numbers. Let an even function $\vartheta, \eta, \psi: X \rightarrow Y$ satisfies the inequality

$$
\|\vartheta(2 p)-48 \vartheta(p)-16 \vartheta(-p)\| \leq\left\{\begin{array}{l}
\lambda, \\
\lambda\|p\|^{s}, \quad s \neq 6 ;
\end{array}\right.
$$

$$
\|\eta(2 q)-192 \eta(q)-64 \eta(-q)\| \leq\left\{\begin{array}{l}
\lambda, \\
\lambda\|q\|^{s}, \quad s \neq 8 ;
\end{array}\right.
$$

$$
\|\psi(2 r)-768 \psi(r)-256 \psi(-r)\| \leq\left\{\begin{array}{l}
\lambda, \\
\lambda\|r\|^{s}, \quad s \neq 10 ;
\end{array}\right.
$$

for all $p, q, r \in X$. Then there exists a unique quintic,septic,nonic mapping $S_{7}, O_{8}, D_{10}: X \rightarrow Y$ such that

$$
\begin{aligned}
& \left\|\vartheta(p)-S_{7}(p)\right\| \leq\left\{\begin{array}{l}
\frac{\lambda}{63}, \\
\frac{\lambda|| p||^{s}}{\left|2^{6}-2^{s}\right|},
\end{array}\right. \\
& \left\|\eta(p)-O_{8}(q)\right\| \leq\left\{\begin{array}{l}
\frac{\lambda}{253}, \\
\frac{\left.\lambda|| p\right|^{s}}{\left|2^{8}-2^{s}\right|},
\end{array}\right. \\
& \left\|\psi(r)-D_{10}(r)\right\| \leq\left\{\begin{array}{l}
\frac{\lambda}{1023}, \\
\frac{\lambda|| p||^{s}}{\left|2^{10}-2^{s}\right|},
\end{array}\right.
\end{aligned}
$$

for all $p, q, r \in X$. 


\section{Stability Results: Mixed Case}

In this section, the generalized Ulam-Hyers stability of the functional equation (1.3), (1.4) and (1.5) mixed case is proved by using fixed point method.

Theorem 7.1. Let $\vartheta, \eta, \psi: X \rightarrow Y$ be a mapping for which there exist a function $\xi, \zeta, \varsigma: X \rightarrow[0, \infty)$ with the condition (5.1), (5.2) and (5.3); (6.1), (6.2) and (6.3) where $\mu_{i}=2$ if $i=0$ and $\mu_{i}=\frac{1}{2}$ if $i=1$ such that the functional inequality with

$$
\begin{aligned}
& \|\vartheta(2 p)-48 \vartheta(p)-16 \vartheta(-p)\| \leq \xi(p) \\
& \|\eta(2 q)-192 \eta(q)-64 \eta(-q)\| \leq \zeta(q) \\
& \|\psi(2 r)-768 \psi(r)-256 \psi(-r)\| \leq \varsigma(r)
\end{aligned}
$$

for all $x, y, z \in X$. If there exists $L=L(i)<1$ such that the function

$$
\begin{aligned}
& p \rightarrow \beta(p)=\xi\left(\frac{p}{2}\right), \\
& q \rightarrow \beta(q)=\zeta\left(\frac{q}{2}\right), \\
& r \rightarrow \beta(r)=\varsigma\left(\frac{r}{2}\right),
\end{aligned}
$$

has the properties (5.7),(5.8) and (5.9); (6.7),(6.8) and (6.9); for all $p, q, r \in X$. Then there exists a unique quintic,septic,nonic mapping $Q_{5}, S_{7}, N_{9}: X \rightarrow Y$ and a unique sextic,octic,decic mapping $S_{6}, O_{8}, D_{10}: X \rightarrow Y$ such that

$$
\begin{gathered}
\left\|\vartheta(p)-Q_{5}(p)-S_{6}(p)\right\| \leq \frac{L^{1-i}}{1-L}(\beta(p)+\beta(-p)) \\
\left\|\eta(q)-S_{7}(q)-O_{8}(q)\right\| \leq \frac{L^{1-i}}{1-L}(\beta(q)+\beta(-q)) \\
\left\|\psi(r)-N_{9}(r)-D_{10}(r)\right\| \leq \frac{L^{1-i}}{1-L}(\beta(r)+\beta(-r))
\end{gathered}
$$

holds for all $p, q, r \in X$.

Proof. Let

$$
\begin{aligned}
& \vartheta_{o}(p)=\frac{\vartheta(p)-\vartheta(-p)}{2} \\
& \eta_{o}(q)=\frac{\eta(q)-\eta(-q)}{2} \\
& \psi_{o}(r)=\frac{\psi(r)-\psi(-r)}{2}
\end{aligned}
$$

for all $p, q, r \in X$. Then $\vartheta_{o}(0)=0, \eta_{o}(0)=0$ and $\psi_{o}(0)=0$ and $\vartheta_{o}(-p)=-\vartheta_{o}(p), \eta_{o}(-q)=-\eta_{o}(q), \psi_{o}(-r)=-\psi_{o}(r)$ for all $p, q, r \in X$. Hence

$$
\begin{gathered}
\left\|\vartheta_{0}(2 p)-48 \vartheta_{o}(p)-16 \vartheta_{o}(-p)\right\| \leq \frac{\xi(p)}{2}+\frac{\xi(-p)}{2} \\
\left\|\eta_{o}(2 q)-192 \eta_{o}(q)-64 \eta_{o}(-q)\right\| \leq \frac{\zeta(q)}{2}+\frac{\zeta(-q)}{2} \\
\left\|\psi_{o}(2 r)-768 \psi_{o}(r)-256 \psi_{o}(-r)\right\| \leq \frac{\varsigma(q)}{2}+\frac{\varsigma(-q)}{2}
\end{gathered}
$$

for all $p, q, r \in X$. By Theorem 5.1, we have

$$
\begin{gathered}
\left\|\vartheta(p)-Q_{5}(p)\right\| \leq \frac{1}{2}\left[\frac{L^{1-i}}{1-L}(\beta(p)+\beta(-p))\right] \\
\left\|\eta(q)-S_{7}(q)\right\| \leq \frac{1}{2}\left[\frac{L^{1-i}}{1-L}(\beta(q)+\beta(-q))\right] \\
\left\|\psi(r)-N_{9}(r)\right\| \leq \frac{1}{2}\left[\frac{L^{1-i}}{1-L}(\beta(r)+\beta(-r))\right]
\end{gathered}
$$

for all $p, q, r \in X$. Also, Let

$$
\begin{aligned}
& \vartheta_{e}(p)=\frac{\vartheta(p)+\vartheta(-p)}{2} \\
& \eta_{e}(q)=\frac{\eta(q)+\eta(-q)}{2} \\
& \psi_{e}(r)=\frac{\psi(r)+\psi(-r)}{2}
\end{aligned}
$$

for all $p, q, r \in X$. Then $\vartheta_{e}(0)=0, \eta_{e}(0)=0$ and $\psi_{e}(0)=0$ and $\vartheta_{e}(-p)=\vartheta_{e}(p), \eta_{e}(-q)=\eta_{e}(q), \psi_{e}(-r)=\psi_{e}(r)$ for all $p, q, r \in X$. Hence

$$
\left\|\vartheta_{e}(2 p)-48 \vartheta_{e}(p)-16 \vartheta_{e}(-p)\right\| \leq \frac{\xi(p)}{2}+\frac{\xi(-p)}{2}
$$

$$
\begin{aligned}
& \left\|\eta_{e}(2 q)-192 \eta_{e}(q)-64 \eta_{e}(-q)\right\| \leq \frac{\zeta(q)}{2}+\frac{\zeta(-q)}{2} \\
& \left\|\psi_{e}(2 r)-768 \psi_{e}(r)-256 \psi_{e}(-r)\right\| \leq \frac{\varsigma(r)}{2}+\frac{\varsigma(-r)}{2}
\end{aligned}
$$


for all $p, q, r \in X$. By Theorem 6.1, we have

$$
\begin{aligned}
& \left\|\vartheta(p)-S_{6}(p)\right\| \leq \frac{1}{2}\left[\frac{L^{1-i}}{1-L}(\beta(p)+\beta(-p))\right] \\
& \left\|\eta(q)-O_{8}(q)\right\| \leq \frac{1}{2}\left[\frac{L^{1-i}}{1-L}(\beta(q)+\beta(-q))\right] \\
& \left\|\psi(r)-D_{10}(r)\right\| \leq \frac{1}{2}\left[\frac{L^{1-i}}{1-L}(\beta(r)+\beta(-r))\right]
\end{aligned}
$$

for all $p, q, r \in X$. Define

$$
\begin{aligned}
& \vartheta(p)=\vartheta_{e}(p)+\vartheta_{o}(p) \\
& \vartheta(q)=\vartheta_{e}(q)+\vartheta_{o}(q) \\
& \vartheta(r)=\vartheta_{e}(r)+\vartheta_{o}(r)
\end{aligned}
$$

for all $p, q, r \in X$. From (7.13), (7.14) and (7.15); (7.16), (7.17) and (7.18); (7.19) (7.20) and (7.21) we arrive

$$
\begin{aligned}
& \left\|\vartheta(p)-Q_{5}(p)-S_{6}(p)\right\| \\
& =\left\|\vartheta_{e}(p)+\vartheta_{o}(p)-Q_{5}(p)-S_{6}(p)\right\| \\
& \leq\left\|\vartheta_{o}(p)-Q_{5}(p)\right\|+\left\|\vartheta_{e}(p)-S_{6}(p)\right\| \\
& \leq \frac{1}{2}\left[\frac{L^{1-i}}{1-L}(\beta(p)+\beta(-p))+\frac{1}{2} \frac{L^{1-i}}{1-L}(\beta(p)+\beta(-p))\right] \\
& \leq \frac{L^{1-i}}{1-L}(\beta(p)+\beta(-p)) \\
& \left\|\eta(q)-S_{7}(q)-O_{8}(q)\right\| \\
& =\left\|\eta_{e}(q)+\eta_{o}(q)-S_{7}(q)-O_{8}(q)\right\| \\
& \leq\left\|\eta_{o}(q)-S_{7}(q)\right\|+\left\|\eta_{e}(q)-O_{8}(q)\right\| \\
& \leq \frac{1}{2}\left[\frac{L^{1-i}}{1-L}(\beta(q)+\beta(-q))+\frac{1}{2} \frac{L^{1-i}}{1-L}(\beta(q)+\beta(-q))\right] \\
& \leq \frac{L^{1-i}}{1-L}(\beta(q)+\beta(-q)) \\
& \leq \frac{L^{1-i}}{1-L}(\beta(r)+\beta(-r)) \\
& \left\|\psi(r)-N_{9}(r)-D_{10}(r)\right\| \\
& =\left\|\psi_{e}(r)+\psi_{o}(r)-N_{9}(r)-D_{10}(r)\right\| \\
& \leq\left\|\psi_{o}(r)-N_{9}(r)\right\|+\left\|\psi_{e}(r)-D_{10}(r)\right\| \\
& \leq \frac{1}{2}\left[\frac{L^{1-i}}{1-L}(\beta(r)+\beta(-r))+\frac{1}{2} \frac{L^{1-i}}{1-L}(\beta(r)+\beta(-r))\right] \\
& \\
& \leq L^{-}(\beta)
\end{aligned}
$$

for all $p, q, r \in X$. Hence the theorem is proved.
Using Corollaries 5.2 and 6.2, we have the following Corollary concerning the stability of (1.3), (1.4), and (1.5).

Corollary 7.2. Let $\lambda$ and $s$ be nonnegative real numbers. Let an even function $\vartheta, \eta, \psi: X \rightarrow Y$ satisfies the inequality

$$
\begin{aligned}
& \|\vartheta(2 p)-48 \vartheta(p)-16 \vartheta(-p)\| \leq \begin{cases}\lambda, & \\
\lambda\|p\|^{s}, & s \neq 5,6 ; \\
(7.22)\end{cases} \\
& \|\eta(2 q)-192 \eta(q)-64 \eta(-q)\| \leq \begin{cases}\lambda, \\
\lambda\|q\|^{s}, & s \neq 7,8\end{cases}
\end{aligned}
$$

$$
\|\psi(2 r)-768 \psi(r)-256 \psi(-r)\| \leq\left\{\begin{array}{l}
\lambda, \\
\lambda\|r\|^{s},
\end{array}\right.
$$

for all $p, q, r \in X$. Then there exists a unique quintic,septic,nonic mapping $Q_{5}, S_{7}, N_{9}: X \rightarrow Y$ and a unique sextic,octic,decic mapping $S_{6}, O_{8}, D_{10}: X \rightarrow Y$ such that

$$
\left\|\vartheta(p)-Q_{5}(p)-S_{6}(p)\right\| \leq\left\{\begin{array}{l}
\left(\frac{\lambda}{31}+\frac{\lambda}{63}\right) \\
\frac{\lambda \| p||^{s}}{\left|2^{5}-2^{s}\right|}+\frac{\lambda\|p\|^{s}}{\left|2^{6}-2^{s}\right|}
\end{array}\right.
$$

$$
\left\|\eta(q)-S_{7}(q)-O_{8}(q)\right\| \leq\left\{\begin{array}{l}
\left(\frac{\lambda}{127}+\frac{\lambda}{253}\right) \\
\left(\frac{\lambda \| p||^{s}}{\left|2^{7}-2^{s}\right|}+\frac{\lambda\|p\|^{s}}{\left|2^{8}-2^{s}\right|}\right.
\end{array}\right)
$$

$$
\left\|\psi(r)-N_{9}(r)-D_{10}(r)\right\| \leq\left\{\begin{array}{l}
\left(\frac{\lambda}{511}+\frac{\lambda}{1023}\right) \\
\frac{\lambda\|r\|^{s}}{\left|2^{9}-2^{s}\right|}+\frac{\lambda\|r\|^{s}}{\left|2^{10}-2^{s}\right|}
\end{array}\right)
$$

for all $p, q, r \in X$.

\section{References}

[1] J. Aczel and J. Dhombres, Functional Equations in Several Variables, Cambridge Univ, Press, 1989.

[2] T. Aoki, On the stability of the linear transformation in Banach spaces, J. Math. Soc. Japan, 2 (1950), 64-66.

[3] M. Arunkumar, A. Bodaghi, J. M. Rassias, E. Sathya, The general Solution and approximations of a Decic type functional equation in various normed spaces, Journal of the Chungcheong Mathematical Society, Vol. 29, No. 2 (2016), 287-328 
[4] M. Arunkumar, P. Agilan,Fixed point stability of a $A Q$ functional equation in RN space, Proceedings of National Conference on Pure and Applied Mathematics, (2014), 37-44, ISBN: 978-93-83459-46-9.

[5] M. Arunkumar, P. Agilan, Stability of a AQC Functional Equation in Fuzzy Normed Spaces: Direct Method, Jamal Academic Research Journal an Interdisciplinary, (2015), 78-86 .

[6] M. Arunkumar, P. Agilan, N. Mahesh kumar,UlamHyers stability of $a r_{i}$ type $n$ dimensional additive quadratic functional equation in quasi beta normed spaces: a fixed point approach, Malaya Journal of Mathematics, (2015), 192 - 202.

[7] M. Arunkumar, P. Agilan, Solution and Ulam-Hyers stability of a $r_{i}$ type $n$ dimensional additive quadratic functional equation in quasi beta normed spaces, Malaya Journal of Mathematics, (2015), 203 - 214.

[8] M. Arunkumar, P. Agilan, C. Devi Shyamala Mary, Permanence of a Generalized AQ Functional Equation In Quasi-Beta Normed Spaces, International Journal of Pure and Applied Mathematics, Vol. 101, No. 6 (2015), 1013- 1025.

[9] John M. Rassias, M. Arunkumar, P. Agilan, Solution and Ulam - Hyers stability of an additive - quadratic functional equation in Banach Space: Hyers Direct and Fixed Point Methods, International Journal of Mathematics and its Applications, Volume 3, Issue 4 - D (2015), 17-46.

[10] S. Czerwik, Functional Equations and Inequalities in Several Variables, World Scientific, River Edge, NJ, 2002.

[11] G. Z. Eskandani, P. Găvrută, J. M. Rassias, R. Zarghami, Generalized Hyers-Ulam Stability for a General Mixed Functional Equation in Quasi- $\beta$-normed Spaces, Mediterr. J. Math. 8 (2011), 331-348

[12] G. Z. Eskandani, P. Găvrută,On the stability problem in quasi-Banach spaces, Nonlinear Funct. Anal. Appl. (to appear).

[13] P. Găvrută, A generalization of the Hyers-Ulam-Rassias stability of approximately additive mappings, J. Math. Anal. Appl., 184 (1994), 431-436.

[14] P. Găvrută, An answer to a question of J.M.Rassias concerning the stability of Cauchy functional equation, Advances in Equations and Inequalities, Hadronic Math. Ser., (1999), 67-71.

[15] P. Găvrută, On a problem of G. Isac and Th. M. Rassias concerning the stability of mappings, J. Math. Anal. Appl. 261 (2001), 543-553.

[16] D. H. Hyers, On the stability of the linear functional equation, Proc.Nat. Acad.Sci.,U.S.A.,27 (1941) 222-224.

[17] D. H. Hyers, G. Isac, Th. M. Rassias, Stability of functional equations in several variables, Birkhauser, Basel, 1998.

[18] S. M. Jung, Hyers-Ulam-Rassias Stability of Functional Equations in Mathematical Analysis, Hadronic Press,
Palm Harbor, 2001.

[19] D. O. Lee, Hyers-Ulam stability of an addtiive type functional equation, J. Appl. Math. and Computing, 13 (2003) no.1-2, 471-477.

[20] B. Margoils and J. B. Diaz, A fixed point theorem of the alternative for contractions on a generalized complete metric space, Bull. Amer. Math. Soc., 126(74) (1968), 305-309.

[21] John M. Rassias, M. Arunkumar, E. Sathya, T. Namachivayam, Various Generalized Ulam - Hyers Stabilities of a Nonic Functional Equation, Tbilisi Mathematical Journal, 9(1) (2016), pp. 159 - 196.

[22] J. M. Rassias, On approximately of approximately linear mappings by linear mappings, J. Funct. Anal. USA, 46, (1982) 126-130.

[23] J. M. Rassias, H. M. Kim, Generalized Hyers-Ulam stability for general additive functional equations in quasi$\beta$-normed spaces. J. Math. Anal. Appl., 356, (2009), 302-309.

[24] J. M. Rassias, K. W. Jun, H. M. Kim, Approximate $(m, n)$-Cauchy - Jensen Additive Mappings in $C^{*}$ algebras, Acta Mathematica Sinica, English Series, Vol. 27, No. 10, (2011), 1907-1922.

[25] K. Ravi, M. Arunkumar, On a n-dimensional additive Functional Equation with fixed point Alternative, Proceedings of International Conference on Mathematical Sciences 2007, Malaysia.

[26] K. Ravi, M. Arunkumar and J. M. Rassias, On the Ulam stability for the orthogonally general EulerLagrange type functional equation, International Journal of Mathematical Sciences, Autumn 2008 Vol.3, No. 08, $36-47$.

[27] Th. M. Rassias, On the stability of the linear mapping in Banach spaces, Proc.Amer.Math. Soc., 72 (1978), 297300.

[28] S. M. Ulam, Problems in Modern Mathematics, Science Editions, Wiley, NewYork, 1964 (Chapter VI, Some Questions in Analysis: 1, Stability).

[29] T. Z. Xu, J. M. Rassias, Approximate Septic and Octic mappings in quasi- $\beta$-normed spaces, J. Comput. Anal. Appl., 15, No. 6 (2013), 1110- 1119.

[30] T.Z. Xu, J.M. Rassias, M. J. Rassias, W. X. Xu, A fixed point approach to the stability of quintic and sextic functional equations in quasi- $\beta$-normed spaces, J. Inequal. Appl. 2010, Art. ID 423231, 23 pp.

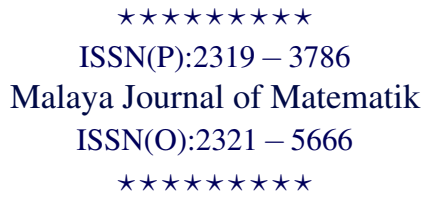

